\title{
Confined longitudinal acoustic phonon modes in free-standing Si membranes coherently excited by femtosecond laser pulses
}

\author{
Florian Hudert, Axel Bruchhausen, Daniel Issenmann, Olivier Schecker, \\ Reimar Waitz, Artur Erbe, Elke Scheer, and Thomas Dekorsy \\ Department of Physics and Center of Applied Photonics, Universität Konstanz, D-78457 Konstanz, Germany
}

Adnen Mlayah

Centre d'Elaboration de Matériaux et d'Etudes Structurales CEMES-CNRS, Université de Toulouse, 29 Rue Jeanne Marvig, 31055 Toulouse, France

Jean-Roch Huntzinger

GES-UMR 5650, CNRS, Université Montpellier 2, 34695 Montpellier Cedex 5, France

(Received 17 March 2009; revised manuscript received 4 May 2009; published 19 May 2009)

\begin{abstract}
In this Rapid Communication we report the first time-resolved measurements of confined acoustic phonon modes in free-standing Si membranes excited by fs laser pulses. Pump-probe experiments using asynchronous optical sampling reveal the impulsive excitation of discrete acoustic modes up to the 19th harmonic order for membranes of two different thicknesses. The modulation of the membrane thickness is measured with fm resolution. The experimental results are compared with a theoretical model including the electronic deformation potential and thermal stress for the generation mechanism. The detection is modeled by the photoelastic effect and the thickness modulation of the membrane, which is shown to dominate the detection process. The lifetime of the acoustic modes is found to be at least a factor of 4 larger than that expected for bulk Si.
\end{abstract}

DOI: $10.1103 /$ PhysRevB.79.201307

PACS number(s): 78.66.Db, 62.25.Jk, 63.22.Dc, 78.47.jc

Free-standing thin semiconductor membranes have a wide range of applications, for example, as key elements in nanomechanical systems, ${ }^{1}$ sensors, ${ }^{2}$ or optomechanical systems. ${ }^{3}$ Thorough understanding of the mechanical and elastic properties in these structures is crucial for the design and engineering of the desired performance of these potential devices. ${ }^{4}$ When the dimension of these structures is reduced to the order of magnitude of the phonon wavelength, the confinement of acoustic modes leads to a discretization of the acoustic spectrum. These effects have been studied via continuous wave light scattering techniques such as Brillouin and Raman scattering in supported and free-standing thin films. ${ }^{5-9}$ Recently time-resolved experiments have contributed significantly to the understanding of phonon dynamics in nanoscale systems and nanoparticles. ${ }^{4,10-13}$

In this Rapid Communication we present results of femtosecond time-resolved pump-probe experiments performed on free-standing Si membranes with a thickness of a few hundred nanometers. A superposition of oscillations corresponding to frequencies of a fundamental mode and its higher odd harmonics up to the 19th order with lifetimes exceeding $1 \mathrm{~ns}$ is observed in the time domain. The results are successfully modeled using a combined elastic and electromagnetic model. It is shown that the detection process is dominated by the dynamic change in the membrane thickness. Our analysis demonstrates that free-standing Si membranes are a model system, which allows disentanglement of basic phonon-photon interaction processes.

The pump-probe experiments were performed using highspeed asynchronous optical sampling (ASOPS) described in detail before. ${ }^{14}$ Two femtosecond Ti:Sapphire oscillators are used to generate the pump and probe pulses with a duration of less than $100 \mathrm{fs}$. The repetition rates $f_{\text {rep }}$ of about 800
$\mathrm{MHz}$ are stabilized in order to fix the difference of the repetition rates at $\Delta f_{\text {rep }}=10 \mathrm{kHz}$. This offset allows for an automatic scan of the measurement window $\left(f_{\text {rep }}^{-1}=1.2 \mathrm{~ns}\right)$ by the probe pulse in $\Delta f_{\text {rep }}^{-1}=100 \mu$ s without mechanical delay line. The time resolution achieved by this technique lies below 150 fs. Experiments are performed in reflection geometry. The average pump and probe powers did not exceed a total of $110 \mathrm{~mW}$, with typical probe powers of about $10 \mathrm{~mW}$. The spot sizes on the membrane were about $25 \mu \mathrm{m}$ in diameter, and the wavelength used for pump and probe beams was $810 \mathrm{~nm}$. The samples were made from the [100] oriented standard silicon-on-insulator (SOI) wafers produced via the smart cut process. ${ }^{15}$ To obtain the free-standing membrane the substrate of the wafer was etched away. The thermal $\mathrm{SiO}_{2}$ was also removed and only the native oxide remained on the membrane. ${ }^{16}$ Two rectangular membranes (about $1 \times 1 \mathrm{~mm}^{2}$ ) with thicknesses of 221 and $346 \mathrm{~nm}$ were studied.

The acoustic dynamics of a free-standing membrane can be described by the following picture. The small thickness of the membrane leads to a confinement and thus a discretization of the longitudinal acoustic modes much like in an optical resonator. After the excitation of the membrane the boundary conditions given by the free surfaces lead to the formation of standing waves with the relationship between the discrete frequencies $f_{m}$ and the film thickness $d: f_{m}$ $=m v_{s} / 2 d, v_{s}$ is the longitudinal sound velocity, and $m$ $=1,2,3, \ldots$ is an integer denominating the order of the mode.

Figure 1 shows membrane oscillations which have been obtained by subtracting the electronic part of the reflectivity change $\Delta R / R$. Figures 1(a) and 1(b) show the oscillations for the 221-nm (346-nm)-thick sample. In both cases an almost undamped saw-tooth-like characteristic of the transients is 


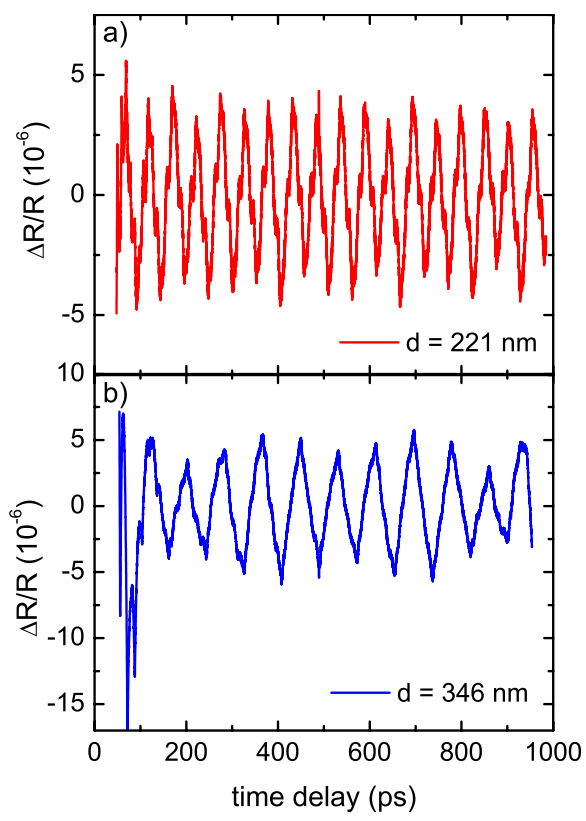

FIG. 1. (Color online) Extracted oscillations from the transients for the (a) $221 \mathrm{~nm}$ sample and (b) the $346 \mathrm{~nm}$ sample. These oscillations have been extracted via subtraction of a multiexponential fit to the original transient.

immediately apparent with the fundamental frequency of the thinner sample being higher than that of the thicker sample. ${ }^{17}$

In Figs. 2(a) and 2(c) the numerical Fourier transformations of the transients are depicted on a log scale. In both cases a series of equidistant peaks with decreasing amplitudes can be observed. A detailed evaluation leads to the conclusion that in both cases the peaks are located at odd harmonics of the fundamental mode at $19.15 \mathrm{GHz}(221 \mathrm{~nm}$
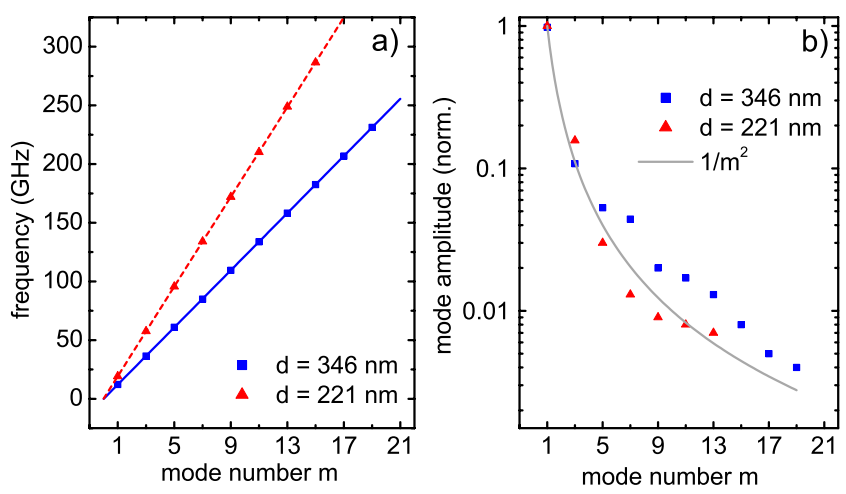

FIG. 3. (Color online) In panel (a) the frequency of the peaks from the measured spectra in Fig. 2 is plotted over the mode number $n$. The respective linear fits for the $221 \mathrm{~nm}$ sample (dotted red line) and the $346 \mathrm{~nm}$ sample (solid blue line) are shown as well. Panel (b) shows the amplitude of the peaks over the mode number $n$. The solid line represents a $1 / \mathrm{m}^{2}$ dependence.

sample) and at $12.17 \mathrm{GHz}$ (346 nm sample). This is evident in Fig. 3(a), where the frequencies of the peaks taken from the spectra are shown as a function of the respective mode number. The values obtained agree very well with the simple picture introduced above. A linear fit yields $19.10 \pm 0.05 \mathrm{GHz}(221 \mathrm{~nm}$ sample) and $12.17 \pm 0.005 \mathrm{GHz}$ (346 nm sample) using a sound velocity of $v_{s}=8430 \mathrm{~m} / \mathrm{s}^{5}$

We compare the experiments to a theoretical model to determine the physical mechanisms involved in the optical excitation and detection steps. The optical excitation of acoustic vibrations in nonpiezoelectric cubic semiconductors arises from the electronic and thermal stresses generated by the pump beam. ${ }^{18}$ After the fast relaxation $(\sim 1 \mathrm{ps})$ of the electron-hole (e-h) plasma, the total stress along the [100] direction is given by ${ }^{19}$
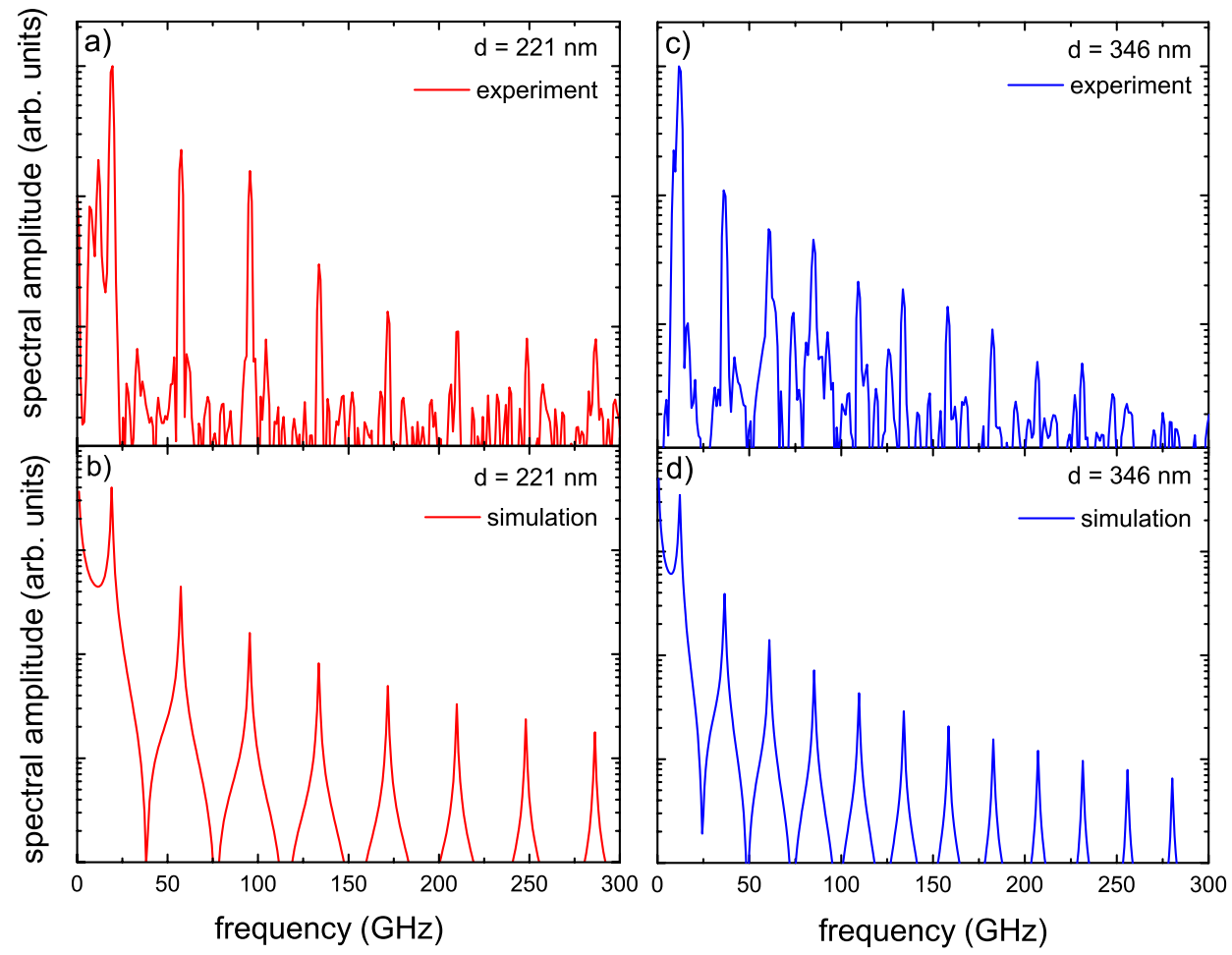

FIG. 2. (Color online) Panels (a) and (c) show the spectra from the respective transients of Fig. 1. In panels (b) and (d) the results from the transfer matrix simulations are shown. 


$$
\sigma=-B \frac{\partial E_{g}}{\partial P} N-3 B \beta \Delta T
$$

where the first term is the electronic contribution $\sigma_{D P}$ arising from the hydrostatic deformation potential $\partial E_{g} / \partial P$. It is proportional to the generated electron-hole pair density $N$ and to the bulk modulus $B$. The second term is the thermal stress $\sigma_{T}$ due to the temperature rise and the subsequent lattice deformation ( $\beta$ being the dilation coefficient). $N$ and $\Delta T$ are determined by the characteristics of the pump beam, and the absorbance $A$ of the membrane

$$
N=\frac{A}{S d} \frac{E_{i}}{h \nu} \quad \text { and } \quad \Delta T=\frac{A}{S d} \frac{E_{i}}{h \nu} \frac{h \nu-E_{g}}{C} .
$$

Here $S$ is the area of the pump beam, $E_{i}$ is the incident energy per pulse, and $h \nu$ is the photon energy of the pump light. $E_{g}$ is the band-gap energy and $C$ is the volumetric heat capacity. Using the physical parameters of bulk silicon ${ }^{20}$ one gets $\sigma_{D P} / \sigma_{T} \sim-7.4$; i.e., the two contributions have different signs and the electronic stress dominates the excitation process. ${ }^{19}$ The large penetration depth of the exciting laser pulse $\left(\alpha^{-1} \sim 8 \mu \mathrm{m}\right)$ (Ref. 20) compared to the membrane thickness and the fast diffusion of the excited carriers leads to a homogeneous carrier distribution within a few 10 ps so that the electronic stress $\sigma_{D P}$ can be considered as homogeneous on the experimental time scale $(1.2 \mathrm{~ns})$.

After a time-domain Fourier transform, the equation of motion for the displacement field of longitudinal acoustic vibrations along the confinement direction $z$ reads as

$$
\frac{\partial^{2} u}{\partial z^{2}}+\left(q^{2}-i \frac{q}{\Lambda}\right) u=0,
$$

where the phonon wave vector $q$ is related to the Fourier angular frequency through: $q=\omega / v_{s}$. In order to avoid divergences of the simulated pump-probe spectra, we included a finite relaxation time $\tau$ for the acoustic vibrations from which the coherence length $\Lambda=v_{s} / \tau$ can be calculated. ${ }^{21}$ A value of $\tau=225$ ps was calculated for room-temperature LA phonon relaxation in bulk silicon. ${ }^{22}$ Using the stress-free boundary conditions at the membrane surfaces, one obtains for the Fourier components of the displacement field

$$
u(z, \omega)=\eta_{0} H(\omega) \frac{\cos (Q z)-\cos [Q(d-z)]}{Q \sin (Q d)},
$$

with

$$
Q^{2}=q^{2}-i \frac{q}{\Lambda} \text { and } \quad \eta_{0}=\frac{-B}{\rho v_{s}^{2}} \frac{\partial E_{g}}{\partial P} N .
$$

$\eta_{0}$ is a dimensionless parameter that describes the deformation amplitude. Since the pump-pulse duration is very short compared to the electron-hole pair lifetime, which is much larger than the $1.2 \mathrm{~ns}$ measurement window (typical values range between $10-100 \mu$ s for crystalline silicon ${ }^{23,24}$ ), the temporal profile of the stress can be described by a Heaviside function, with its Fourier transform being

$$
H(\omega)=\frac{1}{\sqrt{2 \pi}}\left[\pi \delta(\omega)+\frac{i}{\omega}\right] .
$$

The Fourier components of the reflectivity change are evaluated as

$$
d R(\omega)=\left.r^{*} d r\right|_{u=u(\omega)}+r\left(\left.d r\right|_{u=u(\omega)^{*}}\right)^{*},
$$

where $\left.d r\right|_{u=u(\omega)}$ is the difference between the optical reflection coefficient $r$ of the membrane at rest and in the presence of a displacement field $u(\omega)$ given by Eq. (4). This approach is mathematically sound because $r$ is a holomorphous function of the displacement field (in contrast to $R$ ). The reflection coefficients are obtained using the transfer matrix formalism. ${ }^{25}$ Equation (4) is used (i) to determine the modulation of the membrane thickness $\Delta d$ through the displacement of the surfaces as given by $\Delta d=u(z=0)-u(z=d)$ and (ii) to determine the strain field $\eta(z)=\partial u / \partial z$ from which the modulation of the optical index by the photoelastic effect is evaluated as $\delta n=-(3 / 2) n p_{12} \eta \cdot p_{12}$ is the photoelastic coefficient of bulk silicon for modulation by a [100] uniaxial strain. Thus, the thickness of the vibrating optical cavity is modulated and its optical index is nonuniform. These are the two modulation mechanisms considered for the detection process and named optical cavity thickness (OCT) modulation and photoelastic (PE) modulation, respectively. The reflectivity modulation spectra were generated numerically by first subdividing the acoustically deformed membrane into $N$ elementary layers. The overall reflection coefficient is obtained using the transfer matrix formalism. ${ }^{26}$

Lower panels (b) and (d) of Fig. 2 show the Fourier spectra calculated for the two investigated membranes. An analysis of the results of the simulations points out that the contribution of the PE mechanism to the reflectivity modulation is negligible, when compared to the OCT contribution. As a matter of fact we found that, because of the rather small PE coefficient of silicon, the PE contribution is more than 1 order of magnitude smaller than that of the OCT.

As seen in Fig. 2, all calculated peaks correspond to odd acoustic eigenmodes. This can now be understood by evaluating the generation and detection processes. Since we assumed a homogeneous stress, Eq. (4) actually leads to a strong excitation of odd modes. Only a strong stress gradient in the membrane would allow for the efficient generation of even modes. This would occur for example in stronger absorbing membranes (e.g., GaAs) where the generated stress would be inhomogeneous. ${ }^{27}$ But also the detection process is selective because of the dominance of the OCT contribution. Even modes lead to the same displacement on both surfaces so that there is no modulation of the optical cavity thickness. The contribution of odd modes only to the reflectivity modulation is in agreement with our experimental results. It is worth mentioning that from the magnitude of the change in reflectivity this thickness modulation is estimated to be on the order of $10 \mathrm{fm}$, measured with a resolution given by the signal-to-noise ratio of $10^{-7}$ and calculated to be below $1 \mathrm{fm}$.

From the simulations, the Fourier peak intensities decrease as $1 / \omega^{2}$ with increasing frequency. According to Eq. (6) this decay reflects the Fourier transform of the pump beam on the one hand and, on the other hand, the fact that 
shorter wavelength phonons are less excited by the uniform stress. This dependence is reproduced by the experiments as seen in Fig. 3(b). It is worth mentioning that the good agreement with the experiments corroborates the assumption of a uniform distribution of the electronic stress generated by the pump pulse. Indeed, in the case of a nonuniform stress, the decay of the reflectivity modulation peaks with frequency would reflect both the Fourier transform of the pump pulse and the nonuniformity of the stress. Similarly, strong variation in the acoustic coherence length or lifetime with frequency would impact the peak intensities. From the experimental data we cannot determine a strong dependence of the lifetimes on the frequency since all the linewidths are limited by the finite measurement window of $1.2 \mathrm{~ns}$. In contrast the linewidths of the theoretical peaks are given by the assumed bulk LA phonon relaxation time, which leads to a much broader linewidth as is apparent in Fig. 2. This discrepancy points to a larger phonon relaxation time in reduced dimensions by at least a factor of 4 .

In conclusion we have performed pump-probe experiments on free-standing $\mathrm{Si}$ membranes with thicknesses of 221 and $346 \mathrm{~nm}$. Theoretical calculations using a detailed model for the generation and detection processes reveal that the oscillations are probed dominantly through coherent modulation of the membrane thickness and not through the photoelastic effect.

This work is supported by the Deutsche Forschungsgemeinschaft (DFG) through the SFB 767 and by the Ministry of Science, Research and the Arts of Baden Württemberg.
${ }^{1}$ A. Cleland, Foundations of Nanomechanics (Springer, New York, 2003).

${ }^{2}$ W. van Huffelen, M. de Boer, and T. Klapwijk, Appl. Phys. Lett. 58, 2438 (1991).

${ }^{3}$ J. Thompson, B. Zwickl, A. Jayich, F. Marquardt, S. Girvin, and J. Harris, Nature (London) 452, 72 (2008).

${ }^{4}$ D. G. Cahill, W. K. Ford, K. E. Goodson, G. D. Mahan, A. Majumdar, H. J. Maris, R. Merlin, and S. R. Phillpot, J. Appl. Phys. 93, 793 (2003).

${ }^{5}$ J. Groenen, F. Poinsotte, A. Zwick, C. M. Sotomayor-Torres, M. Prunnila, and J. Ahopelto, Phys. Rev. B 77, 045420 (2008).

${ }^{6}$ X. Zhang, R. S. Bandhu, R. Sooryakumar, and B. T. Jonker, Phys. Rev. B 67, 075407 (2003).

${ }^{7}$ X. Zhang, R. Sooryakumar, and K. Bussmann, Phys. Rev. B 68, 115430 (2003).

${ }^{8}$ R. Bhadra, M. Grimsditch, I. K. Schuller, and F. Nizzoli, Phys. Rev. B 39, 12456 (1989).

${ }^{9}$ A. Mlayah, J.-R. Huntzinger, and N. Large, Phys. Rev. B 75, 245303 (2007).

${ }^{10}$ N. Del-Fatti, C. Voisin, F. Chevy, F. Valleé, and C. Flytzanis, J. Chem. Phys. 110, 11484 (1999).

${ }^{11}$ G. A. Antonelli, H. J. Maris, S. G. Malhotra, and J. M. E. Harper, Physica B 316-317, 434 (2002).

${ }^{12}$ R. Taubert, F. Hudert, A. Bartels, F. Merkt, A. Habenicht, P. Leiderer, and T. Dekorsy, New J. Phys. 9, 376 (2007).

${ }^{13}$ W. Huang, W. Qian, and M. El-Sayed, J. Phys. Chem. B 109, 1881 (2005).

${ }^{14}$ A. Bartels, R. Cerna, C. Kistner, A. Thoma, F. Hudert, C. Janke, and T. Dekorsy, Rev. Sci. Instrum. 78, 035107 (2007).

${ }^{15}$ M. Bruel, B. Aspar, and A. J. Auberton-Herve, Jpn. J. Appl. Phys. 36, 1636 (1997)

${ }^{16}$ R. Waitz, O. Schecker, and E. Scheer, Rev. Sci. Instrum. 79,
093901 (2008).

${ }^{17}$ In the first $160 \mathrm{ps}$ of the transients oscillations of higher frequencies are observed, which are damped within 200 ps. Their origin is unclear but may be related to a thin highly strained Si layer. They are subject to further investigations. We would also like to mention that the repetition rate of the pump laser is not commensurate with a subharmonic of the membrane oscillation, which would lead to parametric excitation.

${ }^{18}$ C. Thomsen, H. T. Grahn, H. J. Maris, and J. Tauc, Phys. Rev. B 34, 4129 (1986).

${ }^{19}$ O. Wright and V. Gusev, Appl. Phys. Lett. 66, 1190 (1995).

${ }^{20}$ Physics of Group IV Elements and III-V Compounds, edited by K.-H. Hellwege and O. Madelung, Landolt-Bornstein, New Series, Group III, Vol. 17, Pt. A (Springer, New York, 1982).

${ }^{21}$ Strictly speaking the various acoustic damping mechanisms and their frequency and size dependences should be included in Eq. (3). This is beyond the scope of the present work, and damping is introduced by the single phenomenological parameter $\tau$.

${ }^{22}$ S. Lambade, G. Saharsrabudhe, and S. Rajagopalan, Phys. Rev. B 51, 15861 (1995).

${ }^{23}$ M. S. Tyagi and R. Van Overstraeten, Solid-State Electron. 26, 577 (1983).

${ }^{24}$ Due to the high repetition rate of the pump laser the experiments are performed on a steady-state background of excited e-h pairs.

${ }^{25}$ B. Perrin, in Microscale and Nanoscale Heat Transfer, edited by S. Volz, Topics in Applied Physics, Vol. 107 (Springer, Berlin/ Heidelberg, 2007), p. 333.

${ }^{26}$ After a convergence check $N=201$ has been used in the calculations.

${ }^{27}$ P. Babilotte, E. Morozov, P. Ruello, D. Mounier, M. Edely, J. Breteau, A. Bulou, and V. Gusev, J. Phys.: Conf. Ser. 92, 012019 (2007) 